\title{
Affordances, adaptive tool use and grounded cognition
}

\author{
Antonello Pellicano ${ }^{1 *}$, Serge Thill ${ }^{2}$, Tom Ziemke ${ }^{2}$ and Ferdinand Binkofski ${ }^{1}$ \\ Section of Neurological Cognition Research, Department of Neurology, University Hospital RWTH Aachen, Aachen, North Rhine-Westphalia, Germany \\ 2 School of Humanities and Informatics, University of Skövde, Skövde, Sweden \\ *Correspondence: apellicano@ukaachen.de
}

\section{A commentary on}

The neuroscience of storing and molding tool action concepts: how "plastic" is grounded cognition?

by Mizelle, J. C., and Wheaton, L. A. (2010). Front. Psychology 1:195. doi: 10.3389/ fpsyg.2010.00195

\section{PLASTIC OR ADAPTIVE? A NOTE ON TERMINOLOGY}

In our opinion, the use of the term "plasticity" is problematic because it is too generic to properly account for the processes supposed to be involved. Plastic changes in neuronal circuits can reflect either modifications of existing circuits or the generation of new circuits. Thus, when a new action is added to the sensory-motor repertoire of a given tool, it is not possible to demonstrate whether the previous neuronal substrate changed its structure or a new circuit was recruited and connected to the original one. Defining the exact nature of this plasticity is an issue to be addressed in future research. From a behavioral point of view, we would refer to the capability of a concept to attract new motor meanings as "adaptive grounded cognition," thus the higher the number of new actions embedded in one tool concept, the larger is the number of tasks it can be recruited for.

\section{COMMENTS ON IDEOMOTOR APRAXIA}

We believe that both the "pantomime of tool use" and the "selection of the tool appropriate for a task" should be tested on the same patient to allow the model to discriminate between ideomotor and conceptual apraxia.

According to the authors, a patient with ideomotor apraxia can correctly select both the tool and the usage context which are appropriate for a task, but is unable to translate this knowledge into a correct complex movement. Based on their model, the above described deficit can be explained through impairment of the usage context module alone. Patients would correctly select the tool because they identify it visually but would not recall its proper use; consequently, no information can be sent to neurobiomechanics. This would be a more "central" deficit than the authors' example, characterized by a worse performance.

\section{THE ROLE OF AFFORDANCES}

In our opinion the model would be improved if affordance for action mechanisms were integrated: The potentiation of motor interactions consistent with the conventional use of a perceived tool (Bub et al., 2008).

Affordances would easily explain the mechanism underlying the quick choice of the most appropriate tool when an action goal is given. In Figure 1A, bidirectional connections between available tools and usage contexts represent the automatic activation of stable affordances (Borghi and Riggio, 2009) emerging from invariant features of the tool (mainly its functional meaning) incorporated into the object representation in long term memory (e.g., a spoon typically stirs and scoops, a knife cuts and spreads). Activation of stable affordances is independent of context or task to be carried out. Furthermore, for each stable affordance, variable affordances emerging from temporary characteristics of the tools such as their current handle orientation are also activated.

When an action goal is given (stir the coffee), attention is restricted to a specific action context (to stir) and oriented to available and action-consistent tools (spoon and stirrer). Thus, activation of a restricted number of stable and variable affordances is privileged (e.g., "spoon + to stir" and "stirrer + to stir" with the right hand) over those not consistent with the action goal. Here, the controller will select "spoon + to stir" instead of "stirrer + to stir" and execute the required action with the hand corresponding to the handle orientation. Selection between two equally appropriate tools is assumed to be determined by stronger stable and variable affordances being activated.
Unlike the original model, our controller collects sensory-motor feedback from the executed action and sends it to both the potential neurobiomechanics and available tools/usage contexts stages. The first connection allows for on-line corrections of the pre-programmed movement while the second allows for action re-programming should a new tool need to be employed (Figure 1A).

If no canonical tool is available, no stable-variable affordances consistent with "stirring and scooping" and the temporary characteristics of the tool will be activated. However, the presence of an action goal to be accomplished activates the "to stir" usage context (bold framed action) which then calls the controller to scan all available objects and select an alternative with which the required action can be executed.

We also propose more detailed selection criteria. The selection of an object would depend on the amount of structural, semantic, and temporary features it shares with the unavailable canonical tool as a function of the action goal to be accomplished. Thus, to replace the canonical spoon or stirrer the object must, for instance, have a similar structure (elongated and thin), it must be "something that can come into contact with food" and its state must be compatible with the action itself (it cannot be dirty or currently employed in an action incompatible with the required action goal, e.g., spreading mayonnaise). If an object sufficiently matches the features of the unavailable canonical tool, it is selected as a new tool to accomplish the required action via backward connections of the controller with the available tools/usage contexts stages. From the available objects (hammer, knife), the knife is finally selected and a motor program similar to that used for the spoon is implemented (Figure 1B).

In our proposal, the controller stage is therefore crucial in the motor implementation of actions automatically afforded by object perception, and of those voluntarily programmed on new tools. In the first case, 


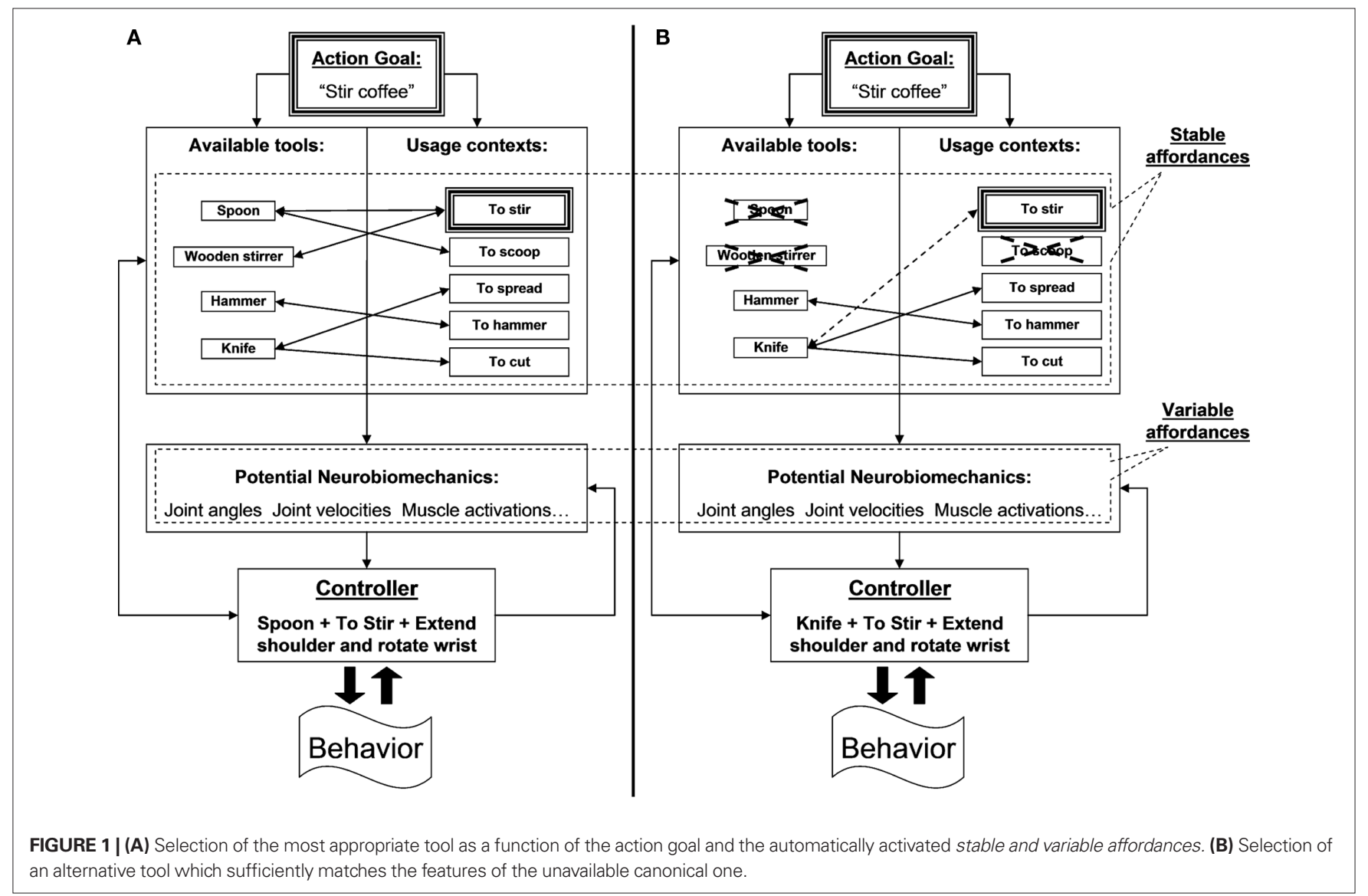

similar to the original model, the controller integrates the product of the activation of available tools, usage contexts, and neurobiomechanic stages (stable and variable affordances) into a defined motor program. In the second case, by receiving information from the action goal/usage context stages, it also governs the selection of a new tool (from the available ones).

The new action-object association is assumed to be very weak and context dependent and to disappear if a canonical object becomes available again. However, if the same action goal is repeated over time in the absence of canonical tools, a new stable affordance will be gained by the originally non-canonical tool. Nevertheless, this new stable functional meaning will remain secondary to the proper canonical use of the tool as well as context dependent.

\section{REFERENCES}

Borghi, A. M., and Riggio, L. (2009). Sentence comprehension and simulation of object temporary, canonical and stable affordances. Brain Res. 1253, 117-128. Bub, D. N., Masson, M. E. J., and Cree, G. S. (2008). Evocation of functional and volumetric gestural knowledge by objects and words. Cognition 106, 27-58.

Received: 17 March 2011; accepted: 22 March 2011; published online: 04 April 2011.

Citation: Pellicano A, Thill S, Ziemke T and Binkofski F (2011) Affordances, adaptive tool use and grounded cognition. Front. Psychology 2:53. doi: 10.3389/fpsyg.2011.00053 This article was submitted to Frontiers in Cognition, a specialty of Frontiers in Psychology.

Copyright (c) 2011 Pellicano, Thill, Ziemke and Binkofski. This is an open-access article subject to a non-exclusive license between the authors and Frontiers Media SA, which permits use, distribution and reproduction in other forums, provided the original authors and source are credited and other Frontiers conditions are complied with. 